\title{
Design of the Automatic Spreader Control System
}

\section{Based on Embedded System}

\author{
Yinglin Li, Lianhe Yang(Correspondence Author) \\ Tianjin Polytechnic University \\ Tianjin 300160, China \\ E-mail: yanglh@tjpu.edu.cn \\ Fang Ma \\ Hebei University of Science and Technology \\ Shijiazhuang 050031, China \\ Yingzhong Li \\ Weifang University of Science and Technology \\ Weifang 262700, China
}

\begin{abstract}
The control system of the traditional automatic spreader with complex structure and inconvenient servicing is designed based on MCU, and the new control system is designed based on the embedded system. Based on the analysis of the work process and work principle for the automatic spreader, we put forward the new scheme to improve the design of the control system for traditional spreader and design the hardware structure and relative software. The new control system can not only actualize the automatic control for the spreader and possess many functions such as spreading tier setup and automatic cloth edge alignment, but also possess the functions including network and system extension and effectively reduce the price of automatic spreader, and the improved spreader will possess stronger functions, more convenient operation and simpler maintenance.
\end{abstract}

Keywords: Computer application, Clothing, Control, Embedded system, Automatic spreader

\section{Introduction}

In the garment production, the sewing processing is implemented after spreading and cutting. The spreading needs 50 200 tiers, and its length is about 5 13 meters, and the human operation needs $4 \sim 6$ people, and the time is about 1 2 hours, and the labor intension is large and the production efficiency is low. It only needs one people to complete the spreading work by the automatic spreader, so the automatic spreader can significantly enhance the production efficiency, effectively reduce the production cost and increase the competition force for clothing enterprises. At present, most automatic spreaders used in China are introduced from foreign countries, and because the price of the equipment is higher, so only few large-sized enterprises can use automatic spreaders. Therefore, it is very meaningful to develop and popularize automatic spreader for such large-sized clothing countries as China.

Because of the limitation of technology, the control system of traditional automatic spreader is actualized by MCU and middle and small sized logic circuit, and the system design is complex, and failures often occur, and the operation speed is low, and the equipment function and the extended ability are worse. In recent years, the quick development of electric technology brings large convenience for the research and development of product, and for example, the price of the 32 bits embedded system only corresponds to the price traditional 8 bits MCU, but the operation ability can achieve thousands MIPS, and the development of the ASIC technology represented by CPLD and FPGA technology (Jia, 2005, P.270-281) largely simplifies the circuit structure, and the operation speed and product performance can be largely enhanced, and at the same time, the ISP technology of the embedded system and ASCI technology can actualize the translation from the hardware design of product to the software design, and largely reduce the research and development cycle of product. In addition, the popularization of the AC servo motor represented by vector control can offer stable base for the exact control of the equipment, and at the same time, with the enhancement of system operation ability, 
many complex control algorithms such as Kalman filter, Fast Fourier Transform (FFT) and fuzzy control have entered into the application stage, which can offer the reliable operation for the system.

\section{Function analysis for control system}

The work of spreader must accord with the requirement of cutting technology, and implement spreading according to the layout length and the planned cutting quantity (Sun, 1994, P.1-3). The automatic spreader should possess functions such as treading, cloth coiling, work state display, cloth edge automatic alignment, automatic rising of cutting knife, cutting, automatic counting and terminal checking, and realize the tension free spreading. So the control system function requirements of spreader should include following aspects.

\subsection{Setup of spreading parameters}

Before spreading, the spreader should set up spreading length, spreading tiers, spreading mode (such as face to face spreading and one way spreading which are seen in Figure 1) and other work parameters according to production requirement, and when the actual spreading length is set up, the length should increase $1 \sim 2 \mathrm{~cm}$ than the plate length obtained through the layout length, and it should be taken as the remnant increment. In the operation, the automatic spreader can automatically compute the spreading length and remnant wastage through the control system, and automatically add up to the needed spreading tiers.

\subsection{Automatic adjustment of tension and edge alignment}

According to the technical requirement, the tension can not occur in the cloth when spreading and the spreader could automatically adjust the tension induced in cloth feeding. In addition, to convenient for cutting, the cloth edges should be automatically realigned.

\subsection{Automatic rise of knife rest}

With the continual increase of spreading tier, the spreading position is higher and higher, and the bracket of the round-bladed cutting knife to support the break could automatic rise.

\subsection{Cloth edge checking}

After one reel cloth is spread, the spreader could check the cloth edge to replace the cloth in time. The spreader could install one reel new tubular cloth on the cloth bracket from the cloth table, and the spreader restarts.

\subsection{Display of work state}

In the spreading process, the spreader could display the evolvements and states of the work such as spreading tier, spreading mode and spreading length.

\section{Hardware design of control system}

The automatic spreader generally includes treading device, cloth loosing device, cloth feeding device, press cloth bracket and other devices, and it is a sort of highly automatic garment equipment, and its hardware structure includes the mechanic part and the control part, and its work principle is seen in Figure 2.

The control system checks the operation state of the equipment through sensors, and controls the electric execution machines of the equipment, and actualizes the automatic operation of the whole equipment.

\subsection{Hardware structure}

To actualize the work requirement of the spreader, we should first implement the structure design of the control system, which mainly control various motors and sensors in the spreader through microprocessor (Chen, 2005, P.39-42). The motors used in the spreader include treading drive motor, cloth loosing drive motor, cloth feeding drive motor, round-bladed cutting drive motor, cutting position drive motor, knife rest lifter driver motor and cloth alignment drive motor, and the sensors include cloth edge alignment sensor, terminal checking sensor and tension checking sensor, and the hardware structure of the control system is seen in Figure 3.

\subsection{Processor selection}

There are many kinds of processor, and their performances and prices have large differences, and proper processor decides the hardware structure of the whole product. At present, ARM9 series embedded processors possess 32 bits operation ability, powerful function and abundant interfaces, and they can realize the functions of the traditional large-sized control system, even workstation based on lower costs (Wayne, 2002, P.30-37). Therefore, the control system of the spreader adopts S3C2410 embedded processor with the kernel of ARM90T. S3C2410 not only has quick operation speed, but offer abundant human-machine interface and conveniently actualize the extension and maintenance interfaces of the equipment.

\subsection{Structure of peripheral circuit}

The main control circuit needs processor and abundant peripheral circuits to realize the whole function (Zhuge, 2003, 
P.104-105), and S3C2410 integrates TFT display interface, touch screen interface, serial interface, USB interface and A/D interface used to sensor sampling. With the development of electric apparatus technology in recent years, the function of CPLD is highly enhanced and the cost reduces with large extents, and the CPLD adopted in the design largely simplifies the circuit design, and both the CPLD and the S3C2410 processor could offer functions such as online diagnosis and JTAC interface, the pin definition of the apparatus, and interior logic, and these functions could be modified in time through the computer, and the circuit design can be largely simplified, and the development process of the circuit will be evolved as software transfer process to overcome the repeat board-projects in the traditional circuit design, and the once design and once modeling of the circuit become possible, which can not only save the research and development costs, but effectively reduce the product research and development circle. The execution motor drive, various relative protections of the equipment, the logical disposals of various circuits and the operation with high timing and reliability in the main circuit of the spreader are realized by CPLD, so the operation speed and reliability of the system can be fully ensured.

\subsection{Equipment interface}

Except for automatic spreading, the spreader also offers convenient human-machine interface and abundant equipment interfaces. The human-machine interface mainly includes 15 inch colorful liquid crystal (TFT), operation keyboard and touch screen. The extension and maintenance interfaces mainly include network interface (ISO 8802.3), serial interface, USB interface and JTAG interface (IEEE 1149.1).

The network interface offers strong extended ability for the equipment, and it can supervise the operation of the equipment and actualize the automatic management in the production through the network, and offer reliable base for the equipment and subsequent extension and update.

The JTAG interface could offer hardware maintenance and updating interface for the equipment, and the JTAG interface could quickly judge the operation state of the hardware system and diagnose the failure of the equipment, and at the same time, the JTAG interface could update the supervision software of the embedded system and the circuit logic of CPLD could complete the function update of the equipment.

\subsection{Selection of execution motor}

The common control motor includes DC motor, stepper motor and AC servo motor. Because of the limitation of brush structure, the DC motor has serious electromagnetic interference and bad reliability. The stepper motor controls simply and could realize certain precision, but the step loss will occur when the opening-loop control of the stepper motor has heavy load, and the mechanic vibration will occur when the speed is low, but the deficient moment will occur when the speed is high. The closed loop control of AC motor has high precision, quick start speed, stable operation and stable interior moment output with rating rev which are ideal performances of the control motor. In the traditional design, the servo motors are rarely adopted because of high costs, and with the development of the control technology, the cost of $\mathrm{AC}$ servo motor quickly reduces, and the AC servo motor tends to replace other control motors, so the motor in the spreader selects AC permanent magnet servo motor which can effectively improve the operation status of the equipment and enhance the equipment efficiency and control precision.

\section{Software design of control system}

The software design of the main control board composed by embedded system CPLD mainly includes operation system collocation, application development and driver development.

\subsection{Collocation of operating system}

The prevalent embedded operating system mainly includes Linux, $\mu$ C/OS- II and Windows CE (Wang, 2002, P.81-124, Li, 2003, P.20-23 \& Li, 2005, P.59-61). The software developed in the Windows CE system has bad stability and complex structure. The Linux system has high opening degree and stable and tidy kernel, so it is the prevalent operating system in present embedded system. According to the demand of spreader control system, we simplify the Linux operating system, leave useful models and cut down useless models. The operating system and application software are stored in the ROM of main control board.

\subsection{Drivers}

The drivers are the extensions of the operating system aiming at concrete hardware, and the hardware is disposed and the standard transfer interface could be offered through drivers. The equipment drivers are integrated in the kernel of the operating system, and they have higher privilege level, memory-resident and sharing. According to the characters of the spreader, the drivers mainly include sensor driver, motor driver, display driver, touch screen driver, network driver, USB driver, serial port driver and electric disk driver.

\subsection{Application}

The application includes equipment operating interface, operation parameter management module, real time control 
module, network management module and communication module (Wang, 2002, P.81-124 \& Huang, 2005, P.44-46). To better organize the software system, we adopt the multiple task work mode, and the spreader is divided into equipment operating management course, control course, network management course and communication course according to concrete application characters. Various courses operate independently and harmonize each other through the information structure. The key technology in the application includes two aspects.

\section{(1) Sensor driver and signal processing}

The sensor translates the physical parameters actually inspected into simulative electric parameters, and translated them into corresponding digital signals through A/D transform. Because the collected signals not only include useful status parameters and various interferences enter into the system together, and these interferences are very harmful to the precision and reliability of the equipment, so we should adopt various measures to eliminate these interferences when the date are collected. The Kalman filter has good filter performance and few signal delay. Various mechanistic resonances and periodic electric interface will occur in the running of spreader, and through the system stability analysis of FFT, we can eliminate system interferences through corresponding measures and ensure that the signals collected by sensors are stable and reliable.

(2) Running control of the equipment

The running control of the equipment includes parameter setup, status display, servo motor driver and dynamic inspection and other modules. We first should set up spreading parameters (including spreading length, spreading speed, spreading tier, spreading mode and remnant increment) according to the requirement of the production technology before spreading. When the tubular cloth loading is completed, the spreader begins to tread, and in the spreading process, the spreader will inspect the cloth edge, cloth tension and cloth edge alignment and adjust the situation in time, and when the tubular cloth uses out, the spreader will automatically stop to replace until to the required spreading tiers. In the spreading process, the spreader could automatically account, and display the spreading tiers and spreading mode real time. The driver of servo motor needs considering the design of the lifter speed and port output, for example, when every tier of cloth begins and ends, the spreader should slowly start and slowly pause, and avoid over leading and lagging.

\subsection{System extended interface}

Because various garment enterprises have different production sizes and products, and they require different equipment functions. To make the equipment better serve for the production, the spreader offers various all-purpose extended interfaces, and through these interfaces, we can conveniently use computer and network resource to extend the equipment functions (Fu, 2005, P.93-97). Except for offering compatible 10BASET standard RJ45 hardware interface, the network interface also could deploy standard network communication agreement and user communication agreement, so users could deploy the system network of production line according to actual situation, and establish the base for the automatic management for the garment enterprises.

The software flow of the control system is seen in Figure 4.

\section{Conclusions}

The control system of automatic spreader using embedded system possesses many advantages such as simple structure, low cost, strong function, high control precision and good extension, and the system has stronger function, more convenient operation and simpler maintenance, and reduces labors' work intension, enhances the rapid reaction ability and the automatization degree of production for the garment enterprises and enhances the competitive ability for enterprises.

\section{References}

Chenfeng. (2005). The Research of the New Fly Frame Control System towards Informationization. Textile Machinery. No.4. P.39-42.

Fuchong, Chenying, Ma, Ximin \& Zhang, Yongyuan. (2005). The Design and Implementation of a General Ethernet Interface for Embedded System. Journal of Shandong University(Engineering Science). No.35(3). P.93-97.

Huang, Jiahong, Zheng, Lingxiang, Zengnan \& Chen, Huihuang. (2005). The Implementation of the Embedded Linux GUI. Journal of Xiamen University(Natural Science). No.44(1). P.44-46.

Jia, Zhiping \& Zhang, Ruihua. (2005). Embedded System Principle and Interface Technology. Beijing: Tsinghua University Press. P.270-281.

Li, Shanping, Liu, Wenfeng \& Wang, Huanlong. (2003). Linux and Embedded System. Beijing: Tsinghua University Press. P.20-23.

Liyong. (2005). Study on the Embedded Linux Integration Development Environment. Journal of Anyang Teachers College. No.6. P.59-61. 
Sun, Surong. (1994). Clothing Machinery Principles and Design. Shanghai: China Textile University Press. P.1-3.

Wang, Tianmiao. (2002). Research and Development Examples on Embedded System Technology: ARM and $\mu$ C/OS- II. Beijing: Tsinghua University Press. P.81-124.

Wayne Wolf. (2002). Exploration of Principle and Method in Design of Embedded Operation System. Beijing: China Machine Press. P.30-37.

Zhuge, Zhenrong \& Liupo. (2003). Applying $\mu$ C/OS-II System to Actualize the Control of Electric Jacquard. Journal of Textile Research. No.24(5). P.104-105.

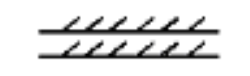

one way spreading

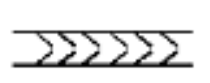

face to face spreading

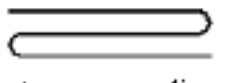

return spreading

Figure 1. Spreading Mode

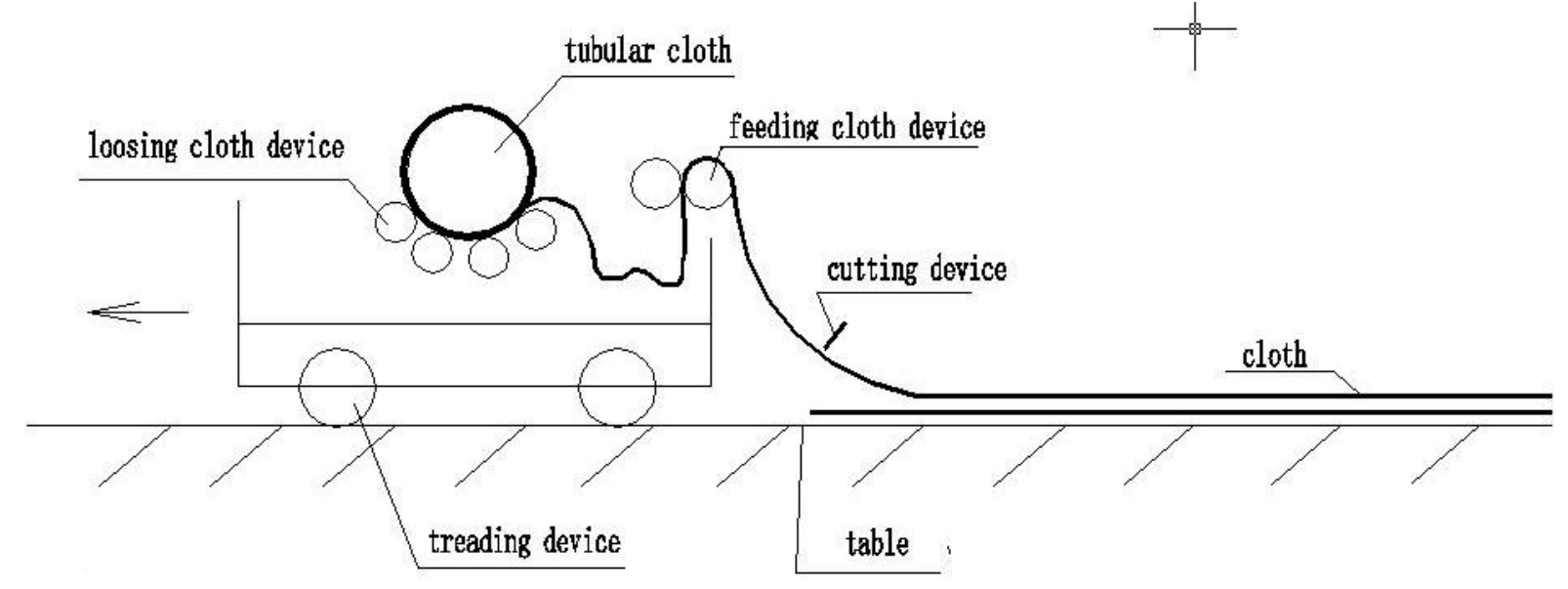

Figure 2. Work Principle of Spreader 


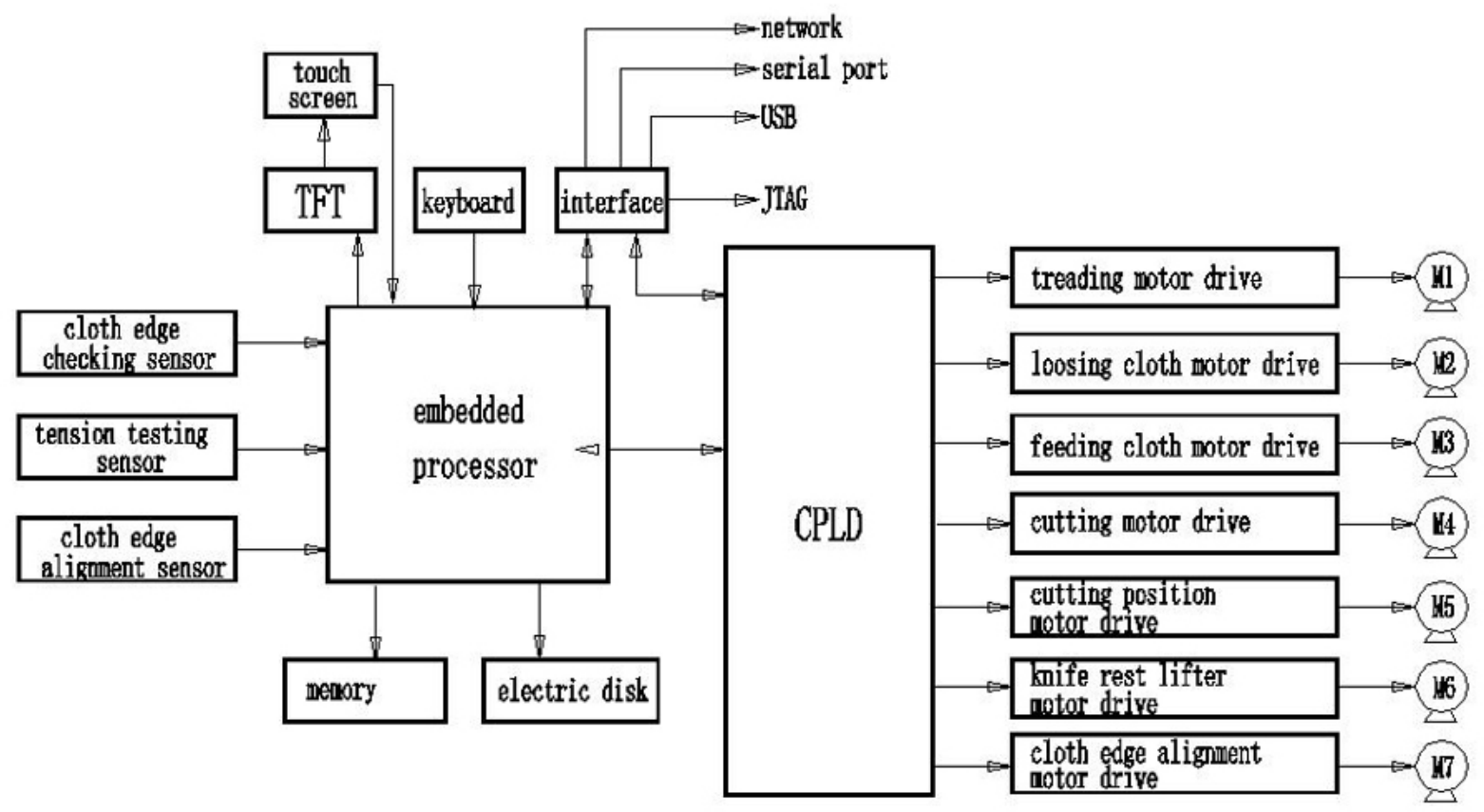

Figure 3. Hardware Structure Chart of Control System 


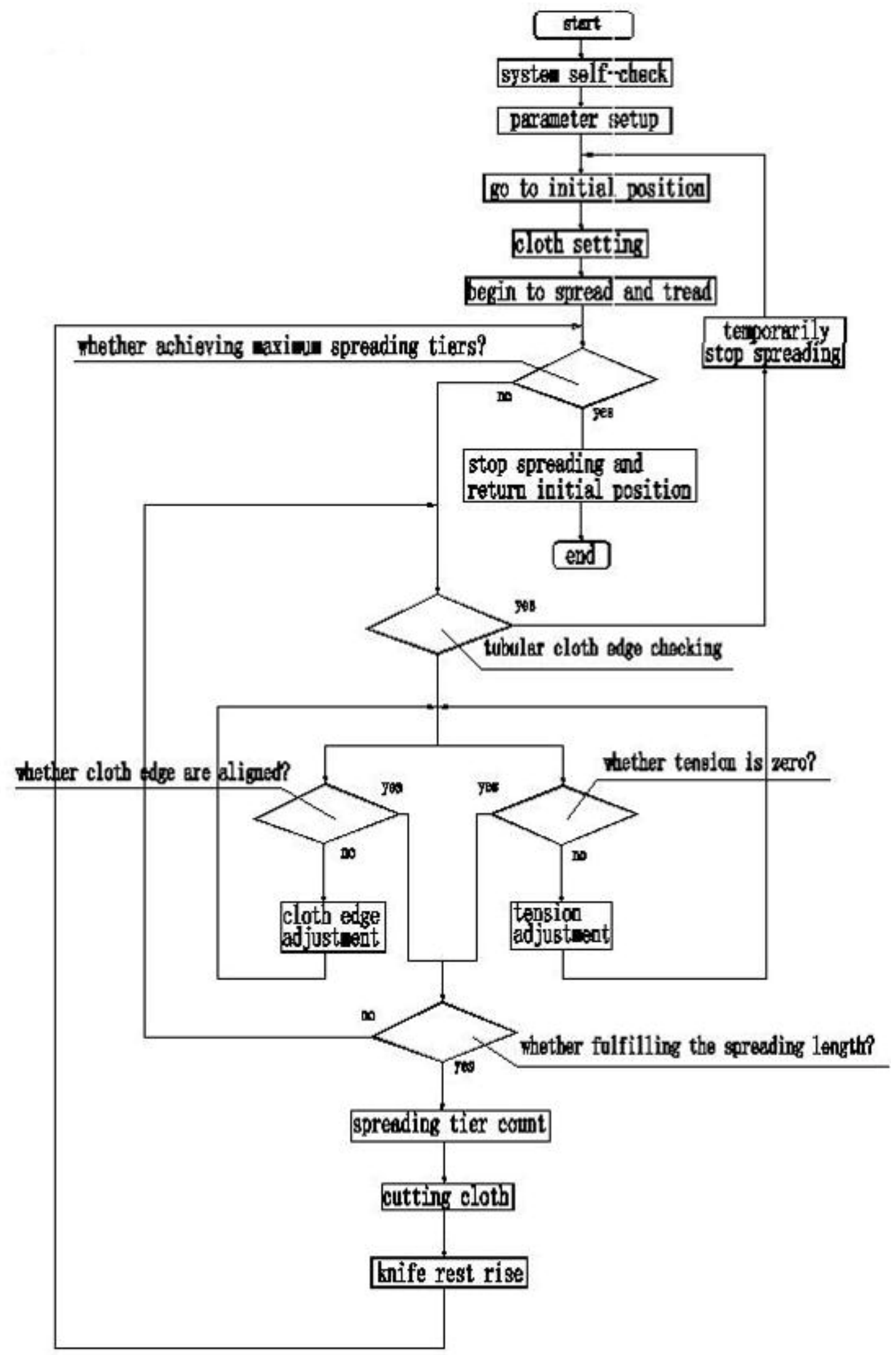

Figure 4. Software Flow Chart of Control System 